УдК 524.7

A. Alexandrov, Ph.D., Seniour researcher, O. Stashko, Post graduate student, V. Zhdanov, Dr.Sci., Prof.,

Astronomical Observatory of Taras Shevchenko National University of Kyiv

\title{
SCALAR FIELD AT LARGE DISTANCES FROM SPHERICALLY SYMMETRIC STATIC CONFIGURATION
}

Stationary spherically symmetric space-time in the quasi-global coordinates is considered in presence of scalar field (SF) minimally coupled to gravity, with a monomial potential $V(\phi)=\phi^{n}, n>4$. We prove convergence of an iterative method to solve the joint system of Einstein - SF equations at sufficiently large distances from the center. The result can be used for a numerical solution for the metric and SF by means of backwards integration from large values of the radial variable to smaller ones.

Keywords: stationary spherically symmetric space-time, scalar field, monomial potential, numerical solution

\section{Introduction}

Considerable attention in gravitational physics is paid to general relativistic configurations with various types of scalar field (SF) (see, e.g., [1,2]). In this field, there are a number of models of the spherically symmetric static configurations with non-linear SF that do not admit exact analytic solutions and, therefore, must be studied numerically. The most direct procedure involves a kind of the backwards problem: we use an approximate solution to derive conditions for ordinary initial value problem at sufficiently large distances $r$ from the configuration and extend this solution by some numerical method for lower $r$. Usually asymptotic solution at spatial infinity is derived "on a physical level of rigor" without necessary justification. We propose such justification that involves a convergent iteration procedure in case of certain SF with monomial potential.

We consider non-linear SF $\phi$ described by the action

$$
S=S_{G R}+\int d^{4} x \sqrt{|g|}\left[\frac{1}{2} g^{\mu v} \phi_{, \mu} \phi_{, v}-V(\phi)\right]
$$

where $S_{G R}$ is the standard gravitational action of General Relativity, $V(\phi)$ is the scalar field self-interaction potential: $V(\phi)=\phi^{n}, n>4$.

Metric of static spherically symmetric space-time in the quasi-global coordinates is

$$
d s^{2}=A(x) d t^{2}-\frac{1}{A(x)} d x^{2}-r^{2}(x)\left(d \theta^{2}+\sin ^{2}(\theta) d \varphi^{2}\right) .
$$

The condition $n>4$ is essential because in this case we expect that $\phi(x) \approx \chi_{0} / x$ on large distances. For $n \leq 4$ the asymptotics is different. The case $n=2$ (linear massive scalar field has been treated in [3]).

The non-trivial Einstein equations following from (1) are

$$
\frac{d}{d x}\left(\frac{d A}{d x} r^{2}\right)=-2 r^{2} V(\phi), \frac{d^{2} r}{d x^{2}}+\frac{1}{2} r\left(\frac{d \phi}{d x}\right)^{2}=0, A \frac{d^{2} r^{2}}{d x^{2}}-r^{2} \frac{d^{2} A}{d x^{2}}=2 .
$$

The equation for SF (covariant D'Alembert equation with the potential $V(\phi)$ in case of static field)

$$
\frac{d}{d x}\left(r^{2} A \frac{d \phi}{d x}\right)=r^{2} \frac{d V}{d \phi}
$$

The equations $(3,4)$ are not independent; further we use the first and the third equations of $(3)$ and equation (4).

\section{Asymptotic conditions and transition to the integral equations}

We consider asymptotic conditions at spatial infinity as follows

$$
A(x)=1-\frac{2 m}{x}+O\left(\frac{1}{x^{2}}\right)
$$

where $m>0$ is the configuration mass,

$$
\begin{gathered}
r^{2}(x)=x^{2}\left(1+\frac{C_{r}}{x}+\frac{D_{r}}{x^{2}}+\ldots\right) \\
\phi(x)=\frac{\chi_{0}}{x}+O\left(\frac{1}{x^{2}}\right)
\end{gathered}
$$

where we put $C_{r}=0$, which is related to the choice of coordinates. In view of these conditions, we can pass to the system of integral equations that can be solved iteratively. Equations (3) yield

$$
\begin{gathered}
r^{2} \frac{d A}{d x}=2 m+2 \int_{x}^{\infty} d x r^{2} V(\phi), \\
A \frac{d r^{2}}{d x}-r^{2} \frac{d A}{d x}=2 x+C
\end{gathered}
$$


In view of asymptotic conditions, the coefficient $C=-6 m$ does not depend on $x_{0}$. Combining these equations we have

where

$$
\begin{aligned}
& r^{2}(x)=\mathbf{R}(x, \phi, r), \\
& A(x)=\mathbf{A}(x, \phi, r), \\
& \phi(x)=\boldsymbol{\Phi}(x, \phi, r),
\end{aligned}
$$

$$
\begin{gathered}
\mathbf{A}(x, \phi, r)=1-\int_{x}^{\infty} d x^{\prime} \frac{2 m+2 \int_{x^{\prime}}^{\infty} d x^{\prime \prime} r^{2} V(\phi)}{r^{2}\left(x^{\prime}\right)}, \\
\mathbf{R}^{2}(x, \phi, r)=r^{2}\left(x_{0}\right)+\int_{x_{0}}^{x} d x^{\prime} \frac{1}{\mathbf{A}(x, \phi, r)}\left[2 x^{\prime}-4 m+2 \int_{x^{\prime}}^{\infty} d x^{\prime \prime} r^{2} V(\phi)\right], \\
\Phi(x, \phi, r)=\int_{x}^{\infty} d x^{\prime} \frac{\chi_{0}+\int_{x^{\prime}}^{\infty} d x^{\prime \prime} r^{2}\left(x^{\prime \prime}\right) \frac{d V}{d \phi}}{R^{2}\left(x^{\prime}\right) A\left(x^{\prime}, \phi, r\right)} .
\end{gathered}
$$

\section{Definition of the class of functions and contraction mapping}

We are looking for the solution $\{r(x), A(x), \phi(x)\}$ of integral equations $(7)$ on $\left[x_{0}, \infty\right)$ with given $\chi_{0}, m>0$ and $r^{2}\left(x_{0}\right)$ satisfying conditions $a x_{0}^{2}<r^{2}\left(x_{0}\right)<b x_{0}^{2}, 0<a=1-\varepsilon<1, \quad b>1$. We fix $x_{0}$ (that affects only the choice of the radial variable); $x_{0}$ must be sufficiently large.

In view of the asymptotic conditions (5), we consider the set $S$ of continuously differentiable functions satisfying for $x \geq x_{0}$

where

$$
\begin{aligned}
& 1-\varepsilon_{1} \leq A(x) \leq 1+\varepsilon_{1}, \\
& \frac{a}{3} x^{2}<r^{2}(x) \leq 3 b x^{2}, \\
& |\phi(x)| \leq \frac{c\left|\chi_{0}\right|}{x}, \quad c>1,
\end{aligned}
$$

$$
0<\varepsilon_{1}<1,1<b\left(1-\varepsilon_{1}\right), c=3+\varepsilon_{2} .
$$

Here $\varepsilon, \varepsilon_{1}, \varepsilon_{2}$ will be assumed to be sufficiently small in view of the asymptotic conditions (4).

For integral equations (7a-c) we have to estimate the r.h.s. of (7) for functions satisfying (8). Now denote $\mathbb{A}=\{\mathbf{A}, \mathbf{R}, \boldsymbol{\Phi}\}-$ operator of the r.h.s. of equations (7). We must show that $A(S) \subset S$ and the mapping $A: S \Rightarrow S$ is a contractor.

After some estimates on account of (8), we have $\left(x \geq x_{0}>>m\right)$

$$
\begin{gathered}
\mathbf{R}^{2}(x, \phi, r)<r^{2}\left(x_{0}\right)+\frac{x^{2}}{1-\varepsilon_{1}}\left[1+6 \frac{b c^{n} \chi_{0}^{n}}{(n-3) x_{0}^{n-2}}\right]<b x^{2}+\frac{x^{2}}{1-\varepsilon_{1}}+\frac{x^{2}}{1-\varepsilon_{1}}\left[6 \frac{b c^{n} \chi_{0}^{n}}{(n-3) x_{0}^{n-2}}\right], \\
\mathbf{R}^{2}(x, \phi, r)>a x_{0}^{2}+\frac{\left(x-x_{0}\right)^{2}}{\left(1+\varepsilon_{1}\right)}-\left(x-x_{0}\right) x_{0}\left[\frac{4 m}{x_{0}}+\frac{2}{1-\varepsilon_{1}} \frac{3 n-5}{n-1} \frac{1}{x_{0}^{n-2}}\left(c \chi_{0}\right)^{n} b\right],
\end{gathered}
$$

whence for a sufficiently large $x_{0}$ we have $a x^{2} / 3<\mathbf{R}^{2}(x, \phi, r) \leq 3 b x^{2}$.

Using (8) we have

$$
|\mathbf{A}(x, \phi, r)-1| \leq \frac{3}{a x_{0}} \cdot\left[2 m+6 \frac{b c^{n} \chi_{0}^{n}}{(n-3) x_{0}^{n-3}}\right],
$$

which is smaller than any prescribed $\varepsilon_{1}$ in case of sufficiently large $x_{0}$. Thus $A(x)$ fulfills (8a).

We shall now estimate $|\Phi|$ using (8)

$$
|\Phi(x, \phi, r)| \leq \frac{3}{x}\left[\left|\chi_{0}\right|+\left.\left|\frac{3 b n}{(n-4) x_{0}^{n-4}}\right| c \chi_{0}\right|^{n-1} \mid\right] .
$$

Then, $|\Phi|$ fulfills $(8 \mathrm{c}, \mathrm{d})$ for $x>x_{0}$ provided that we choose

$$
\left.\left|\frac{9 b n}{(n-4) x_{0}^{n-4}}\right| c \chi_{0}\right|^{n-1} \mid<\varepsilon_{2} ;
$$

this choice being possible for sufficiently large $x_{0}$. 
Thus, the set of functions of $C\left[x_{0}, \infty\right)$ satisfying (8) is invariant with respect to the mapping $A$ defined by the right hand sides of (7).

Now we estimate the contractive properties of $A: S \Rightarrow S$. We denote $\delta f(x) \equiv f(x)-f^{\prime}(x)$, where $f(x), f^{\prime}(x) \in S$.

After cumbersome estimations on account of (8), we get

$$
\left|\mathbf{A}\left(x, \phi^{\prime}, r^{\prime}\right)-\mathbf{A}(x, \phi, r)\right| \leq s_{1}\left\|x^{-2} \delta r^{2}(x)\right\|+s_{2} \chi_{0}^{-1}\|x \delta \phi(x)\|,
$$

where dimensionless $\left.s_{1}=O\left(\frac{m}{x_{0}}\right)+O\left[\left|\chi_{0}\right|^{2}\left(\frac{\chi_{0}}{x_{0}}\right)^{n-2}\right], \quad s_{2}=O\left[\left.|| \chi_{0}\right|^{2}\left(\frac{\chi_{0}}{x_{0}}\right)^{n-2}\right]\right]$ tend to zero for $x_{0} \rightarrow \infty$ (dimensions: $\left.\chi_{0} \sim x ; \quad w \sim x^{-2}\right) ;\|f(x)\| \equiv \sup \left\{|f(x)|, \quad x \geq x_{0}\right\}$.

Next, using (8) we get

$$
x^{-2}\left|\mathbf{R}^{2}\left(x, r^{2}, \Phi\right)-\mathbf{R}^{2}\left(x, r^{\prime 2}, \Phi^{\prime}\right)\right| \leq\left\{s_{3}\left\|x^{-2} \delta r^{2}(x)\right\|+s_{4} \chi_{0}^{-1}\|x \delta \phi(x)\|\right\}
$$

$s_{i}=s_{i}\left(x_{0}\right) \rightarrow 0$ for $x_{0} \rightarrow \infty$.

Analogously for $\Phi(x, \phi, r)$, we get

$$
\begin{gathered}
\left|\Phi(x, \phi, r)-\boldsymbol{\Phi}\left(x, \phi^{\prime}, r^{\prime}\right)\right| \leq \frac{\chi_{0}}{x}\left\{s_{5}\left\|\frac{\delta r^{2}}{x^{2}}\right\|+s_{6} \chi_{0}^{-1}\|x \delta \phi\|\right\} \leq \\
\leq \frac{\chi_{0}}{x} \max \left(s_{5}, s_{6}\right)\left\{\left\|\frac{\delta r^{2}}{x^{2}}\right\|+\chi_{0}^{-1}\|x \delta \phi\|\right\},
\end{gathered}
$$

where $s_{i}=s_{i}\left(x_{0}\right) \rightarrow 0, \quad x_{0} \rightarrow \infty$.

Thus, we see from (10) that the mapping of is contractive in the norm

$$
\|A(x)\|+\left\|\frac{r^{2}(x)}{x^{2}}\right\|+\chi_{0}^{-1}\|x \phi(x)\|,
$$

where $x_{0} \rightarrow \infty$. This finishes the consideration of convergence of the iteration sequence obtained with operator $A$.

By the end, we note that consideration with the norm can be expected to work in much wider situation, e.g., in case of a superposition of several SFs.

Список використаних джерел

1. General Relativity Theory: Recognition through Time / A. N. Alexandrov, I. B. Vavilova, V. I. Zhdanov et al. - K. : Naukova dumka, 2015 (in Russian).

2. Dark energy and dark matter of the universe: in three volumes. Vol. 1. Dark matter: Observational evidence and theoretical models / B. Novosyadlyi, V. Pelykh, Yu. Shtanov, A. Zhuk ; ed. V. Shulga. - K. : Akademperiodyka, 2013

3. Stashko O. S. Spherically symmetric configurations of General Relativity in presence of linear massive scalar field: separation of test body circular orbits distributions / O. S. Stashko, V. I. Zhdanov // Ukr. J. Phys. - 2019. - Vol. 64, № 3 (2019).

Надійшла до редколегії 16.09.19

О. Александров, канд. фіз.-мат. наук, ст. наук. співроб.,

О. Сташко, аспірант,

В. Жданов, д-р фіз.-мат. наук, профр.

Астрономічна обсерваторія

Київського національного університету імені Тараса Шевченка

\section{СКАЛЯРНЕ ПОЛЕ НА ВЕЛИКИХ ВІДСТАНЯХ ВІД СФЕРИЧНО СИМЕТРИЧНОЇ СТАТИЧНОЇ КОНФІГУРАЦІЇ}

Розглянуто нелінійне скалярне поле з дією

$$
S=S_{G R}+\int d^{4} x \sqrt{|g|}\left[\frac{1}{2} g^{\mu v} \phi_{, \mu} \phi_{, v}-V(\phi)\right],
$$

де $S_{G R}$ - стандартна гравітаційна дія загальної теорії відносності, потенціал самодії скалярного поля є мономним: $V(\phi)=\phi^{2 n}$, $n>2$. Умова на степінь $n$ пов'язана із асимптотикою поля на великих відстанях.

Розглянуто сферично симетричні розв'язки системи рівнянь Ейнштейна та скалярного поля у просторі-часі у квазіглобальних координатах із метрикою

$$
d s^{2}=A(x) d t^{2}-A^{-1}(x) d x^{2}-r^{2}(x)\left(d \theta^{2}+\sin ^{2}(\theta) d \varphi^{2}\right) .
$$

Система - це три звичайні диференціальні рівняння; її зведено до інтегральних рівнянь, зручних для проведення ітераційної процедури, яка не виводить із множини $S$ неперервно диференційовних функцій, що задовольняють певні обмеження, зокрема $1-\varepsilon_{1} \leq A(x) \leq 1+\varepsilon_{1}, a x^{2} / 3<r^{2}(x) \leq 3 b x^{2},|\phi(x)| \leq c\left|\chi_{0}\right| / x$, де константи $\varepsilon_{1}, a, b, c, \chi_{0}$ задовольняють певні вимоги. На основі принципу стискаючих відображень показано збіжність ітераційної процедури, яка дає розв'язок на достатньо великих відстанях від центра. Результат може бути використано для числового знаходження метрики та скалярного поля шляхом оберненого інтегрування від великих до менших значень радіальної змінної.

Ключові слова: сферично симетричний простір-час, скалярне поле, мономний потенціал, числове інтегрування 
А. Александров, канд. физ.-мат. наук, ст. науч. сотр.,

А. Сташко, аспирант,

В. Жданов, д-р физ.-мат. наук, профр.

Астрономическая обсерватория

Киевского национального университета имени Тараса Шевченко

\section{СКАЛЯРНОЕ ПОЛЕ НА БОЛЬШИХ РАССТОЯНИЯХ ОТ СФЕРИЧЕСКИ СИММЕТРИЧНОЙ СТАТИЧЕСКОЙ КОНФИГУРАЦИИ}

Рассмотрено сферически симметрическое пространство-время в квазиглобальных координатах в присутствии скалярного поля (Сп), которое минимально связано с гравитацией с мономным потенциалом $V(\varphi)=\varphi^{n}, n>4$. Доказана сходимость итерационной процедуры для решения общей системы уравнений Эйнштейна и скалярного поля на достаточно больших расстояниях от центра. Результат может быть использован для численного нахождения метрики и скалярного поля путём обратного интегрирования от больших до меньших значений радиальной переменной.

Ключевые слова: сферически симметричное пространство-время, скалярное поле, мономный потенциал, численное интегрирование.

удк 524.7

С. Парновський, д-р фіз.-мат. наук, проф.,

І. Ізотова, канд. фріз.-мат. наук,

Київський національний університет імені Тараса Шевченка

\section{ДОСЛІДЖЕННЯ ТЕПЛОВОГО ВИПРОМІНЮВАННЯ В РАДІОКОНТИНУУМІ НА ЧАСТОТІ 1.4 ГГЦ ВИБРАНИХ КОМПАКТНИХ ГАЛАКТИК З АКТИВНИМ ЗОРЕУТВОРЕННЯМ}

Досліджено частки теплового випромінювання у радіоконтинуумі на частоті 1.4 ГГи для підвибірки з 92 компактних галактик з активним зореутворенням, створеної за умови жорсткішого критерію компактності. Як і для повної вибірки зі 193 галактик, отримано, що розподіл частки теплового випромінювання у радіоконтинуумі на частоті 1.4 ГГи близький до лог-нормального з медіанним значенням близько 15 \%; установлено залежність частки від еквівалентної ширини емісійної лінії Нß. Єдина суттєва відмінність полягає в тому, що частка теплового випромінювання для підвибірки з 92 галактик не корелює з індексом кольору $g-r$, як це встановлено для галактик повної вибірки. Обговорюються причини такої відмінності.

Ключові слова: галактики з активним зореутворенням, випромінювання в радіоконтинуумі, теплове випромінювання.

Вступ

Ця робота продовжує дослідження, результати яких детально викладено в наших попередніх статтях $[1,8]$. Вони пов'язані з вивченням випромінювання різних вибірок галактик, у яких перебігають активні процеси зореутворення, у широкому діапазоні довжин хвиль, зокрема в радіоконтинуумі на частоті 1.4 ГГц. Відомо, що це випромінювання переважно має нетеплове походження, але в зонах іонізованого водню його тепловий компонент (free-freeвипромінювання) може бути помітним і розглядатися як опосередкований індикатор процесів зореутворення [5]. Детальний огляд літератури та посилання наведено в роботах $[1,5,6,8]$.

У роботі [8] нами було проаналізовано випромінювання в радіоконтинуумі на частоті 1.4 ГГц 52 галактик з активним зореутворенням з вибірки LCG (Luminous Compact Galaxies), яким притаманні високі світності та еквівалентні ширини EW(Hß) емісійної лінії $\mathrm{H} \beta$. Було показано, що незважаючи на високу активність та відносну молодість спалаху зореутворення в LCG галактиках, про що свідчили значення $E W(H \beta) \geq 100 \AA$, випромінювання в радіоконтинуумі на частоті 1.4 ГГц залишалося переважно нетепловим, а частка $A$ теплового компонента у загальному випромінюванні в радіоконтинуумі становила у середньому $17 \%$.

\section{Вибірка та їі первинне опрацювання}

У роботі [1] було оцінено частку А теплового випромінювання в радіоконтинуумі на частоті 1.4 ГГц для 193 компактних галактик з активним зореутворенням із вибірки CSFG (compact star-forming galaxies) [11], створеної на основі цифрового огляду неба SDSS DR12 [10]. Детальний опис CSFG вибірки та її опрацювання наведено в роботах $[6,11]$. Нагадаємо лише принципові позиції критерію відбору галактик до вибірки: наявність у спектрі емісійних ліній $\mathrm{H} \beta$ з еквівалентною шириною $\mathrm{EW}(\mathrm{H} \beta) \geq 10 \AA$; компактність (розмір $\leq 10$ "); відсутність спектральних ознак наявності

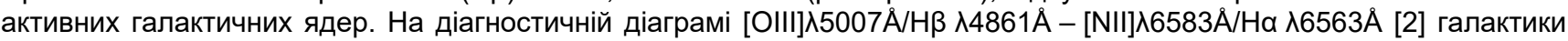
CSFG вибірки займають позиції, притаманні галактикам з активним зореутворенням, а не з активними галактичними ядрами. Галактики для дослідження випромінювання в радіоконтинуумі було відібрано шляхом перехресного ототожнення списку CSFG із переліком радіоджерел каталогів NVSS [12] і FIRST [3] та подальшої візуальної ревізії. Пошук оптичних та радіопартнерів проводився із граничною кутовою відстанню $\leq 15 "$. Для відібраних 193 галактик отримано, що як нижня, так і верхня межі частки $A$ теплового компонента випромінювання в радіоконтинуумі мають лог-нормальні розподіли із середніми значеннями, що відповідають часткам у 5,9 та 14,5 \%, відповідно. Частка зростає при збільшенні еквівалентної ширини $\mathrm{EW}(\mathrm{H} \beta)$ емісійної лінії $\mathrm{H} \beta$ при постійному індексові кольору $g-r(g$ та $r$ - модельні значення зоряної величини у відповідних смугах із бази даних SDSS) або при збільшенні індексу кольору при постійній еквівалентній ширині емісійної лінії Нß.

Про нижню й верхню межі, а не про точні значення частки теплового компонента випромінювання в радіоконтинуумі йдеться з такої причини. За даними про радіовипромінювання було визначено повний потік і світність у радіоконтинуумі на частоті 1.4 ГГц. Електронна температура та електронна густина, що притаманні галактикам із CSFG 